\title{
An Optical Fiber Security System Utilizing Fiber Bragg Grating Sensors
}

\author{
Janw-Wei Wu, Chih-Hsien Shao and Chia-Chin Chiang* \\ Department of Mechanical Engineering, National Kaohsiung University of Applied Sciences, \\ 415 Chien Kung Road, Kaohsiung 807, Taiwan
}

(Received July 2, 2014; accepted April 2, 2015)

Key words: fiber Bragg grating, security systems, strain sensing, optical fiber

In this study, we propose an optical fiber security system based on fiber Bragg grating (FBG) force-loading sensors embedded in the floor for security monitoring. FBG has the advantages of high sensitivity, small size, corrosion resistance, and resistance to electromagnetic interference. Therefore, FBG is very suitable for use as small, highsensitivity sensors for security sensing applications. The system proposed here can monitor the different weights of passing pedestrians through different variations of strain. When thieves attempt a crime, the proposed optical fiber security system is capable of recognizing human body weight and triggering an alarm under the optical fiber security monitoring system. In this study, we utilized FBG sensors bonded to the bottom surface of a floor, and found that when there was a deformation of the floor structure, there was a linear relationship between the wavelength of the FBG sensor and the loading. Therefore, the force loading can be measured by observing the FBG wavelength. The loading sensitivity is about $0.000451 \mathrm{~nm} / \mathrm{N}$, and the R-squared value of the linear relationship reached up to 0.996. Thus, in this study, we establish an accurate and practical assessment technique for security monitoring via packaged FBG sensors that are surface-bonded to the bottom of a floor.

\section{Introduction}

In recent years, owing to growing problems affecting public security, security-related research efforts have gained increasing attention, and many simple and small security systems are starting to be developed. At present, commonly adopted security measures include the use of closed circuit television (CCTV) systems, ${ }^{(1)}$ security guards, fiber sensors, ${ }^{(2)}$ and infrared sensors, ${ }^{(3)}$ among others. For this study, we developed highsensitivity fiber Bragg grating (FBG) sensors that are small, lightweight, and resistant to corrosion and electromagnetic interference. The most common security methods can

${ }^{*}$ Corresponding author: e-mail: ccchiang@kuas.edu.tw 
only cover a specific range and are unable to effectively identify and calculate weight. Therefore, we propose herein an FBG security system that can be used to weigh loads effectively in order to identify different types of objects through the sensing floor. The FBG sensing system has the potential to function as a highly sensitive optical fiber security system.

In 1978, Hill et al. ${ }^{(4)}$ first discovered that an argon ion laser could be used on a photosensitive (Ge-doped) fiber to produce standing-wave periodic structures on the photosensitive fiber, modifying the fiber into the world's first FBG. In 1993, Hill et al. ${ }^{(5)}$ proposed a phase mask in combination with a 248 -nm-wavelength periodic structure produced in the photosensitive optical fiber with an excimer laser. The principle involves using ultraviolet light to produce interference fringes by the phase mask on the grating, so that when the interference fringes shine on the photosensitive fiber, they will cause changes in the refractive index and will thereby form a periodic grating structure. In 2004, Lee et al..$^{(6)}$ proposed a railway axle counter application for FBG sensors, which has the potential to replace the traditional railway axle counting systems.

In the present study, we used a type of FBG also known as a reflection fiber grating that has a grating period of about $1 \mu \mathrm{m}$. When the light transported in the fiber meets the FBG, there will be a specific wavelength of light affected by the fiber grating coupled to the light traveling in reverse. In the present study, we used FBG sensors surface-bonded to the bottom of a hollow floor. When a pedestrian walks on the sensing region, the floor will receive pressure and be deformed. The FBG sensors will, in turn, sense the force loading signal on the floor, and the force loading signal is then transmitted to a computer through analysis software for detection.

\section{Methods}

\subsection{FBG sensor process}

In 1989, Meltz et al. ${ }^{(7)}$ proposed that the FBG be fabricated using an excimer laser $(\lambda=\sim 248 \mathrm{~nm})$ with a frequency of $100 \mathrm{~Hz}$ and a pulse power of $10 \mathrm{~mW}$. The photosensitive fibers (Fibercore PS1550) are used for exposure to side-writing by highenergy UV lasers to form the Bragg grating via the phase mask method. The refractive index of the fiber core generates a periodic change for forming the FBG.

\subsection{Packaging of an FBG sensor}

In this study, polydimethylsiloxane (PDMS) was used for packaging the fiber Bragg grating sensors. PDMS has the advantages of chemical resistance, high temperature stability, high transmittance, excellent flexibility, and low cost. Packaged FBG sensors can be easily surface bonded to a floor in such a way that measurement is unaffected by water or other chemicals. Moreover, PDMS packaging makes an FBG sensor more robust, i.e., it will not break due to mild bending or other impacts. Therefore, a robustly packaged FBG sensor is suitable for use in harsh environments. The PDMS packaging of the FBG sensor is shown in Fig. 1. 


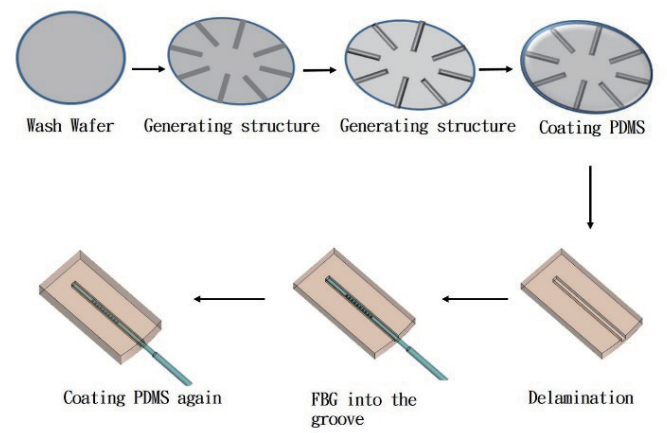

Fig. 1. (Color online) PDMS packaging of FBG sensors.

\subsection{Fundamental principles of FBG}

The FBG sensors employed in this study were fabricated using a UV excimer laser. Laser light was passed through a phase mask to generate interference and form periodic changes in the core refractive index of the photosensitive optical fiber to consequently form optical grating structures. Therefore, this type of grating is also known as a reflective fiber grating. The fiber grating has a grating period of about $1 \mu \mathrm{m}$. When the light emitted from the broadband light source is coupled into the optical fiber, the light reflected by the FBG depends on variations in the periodic refractive index. The light meeting the conditions of the Bragg grating will be reflected, and the remainder of the light will be transmitted. The light that is reflected must have a wavelength meeting the Bragg condition expressed by the following formula: ${ }^{(8)}$

$$
\lambda_{\mathrm{B}}=2 n_{\mathrm{eff}} \Lambda
$$

where $\lambda_{\mathrm{B}}$ represents the Bragg wavelength, $n_{\text {eff }}$ represents the effective refractive index, and $\Lambda$ represents the grating period. If FBG is subjected to force and temperature variations, those variations will affect the grating period, and the changes in the Bragg wavelength will be measured and retrieved from the coupler. Therefore, the reflected light spectrum of FBG can be used as a means for physical quantity measurements by monitoring the FBG wavelength.

\section{Experiment}

FBG sensors were used for the measurement of strain induced by weight load on the floor. The FBG wavelength shift is linear with loading, as calculated according to eq. (1). By observing the FBG wavelength shift, we can obtain the loading variation. The FBG wavelength detection equipment is normally used in an optical spectrum analyzer (OSA), which is limited by the scanning speed. Therefore, we proposed using a high-speed FBG 
interrogation monitor (Ibsen's I-MON) that is built with high-resolution spectrometer technology, utilizing fused silica transmission gratings. This optical analyzer (Ibsen's I-MON) was used to achieve fast measurement frequency for dynamic sensing. The experiment in this study consisted of measuring the frequency of pedestrian pace units of 0.5 $\mathrm{s}$ as various loads were applied to the floor. Loading increased from 392 to $980 \mathrm{~N}$ with $98 \mathrm{~N}$ increments.

The setup of the FBG sensor security alarm system is shown in Fig. 2. Firstly, the broadband light source is transmitted through the coupler to the FBG sensor, then the FBG light signal is reflected back into the coupler and into the FBG wavelength analyzer, which is used for monitoring the FBG wavelength shift. Finally, a computer software program is used for recording data and making alarm determinations. When the sensing signals rise above a certain set value, the alarm system obtains a response signal, and wavelength shifts can be used to identify pedestrian body types, followed by security alarms.

\section{Results and Discussion}

In this study, we investigated the relationship between the load and the optical wavelength shift for an FBG security system. The FBG sensors were affixed to the bottom of a floor, and OSA was used for the observation of FBG spectra. The original wavelength of an FBG sensor without an applied load was $1552.28 \mathrm{~nm}$. Thereafter, the load was increased from 392 to $980 \mathrm{~N}$ in increments of $98 \mathrm{~N}$. Figure 3 shows the spectra of the FBG shifts with various force loadings. The spectra shift from 1552.42 to 1552.68 $\mathrm{nm}$. The total wavelength shift, then, is $0.26 \mathrm{~nm}$, and the relationship between the force loading and the wavelength shift is shown in Fig. 4. The R-squared value of the linear relationship reached up to 0.996 , and the loading sensitivity was about $0.000451 \mathrm{~nm} / \mathrm{N}$,

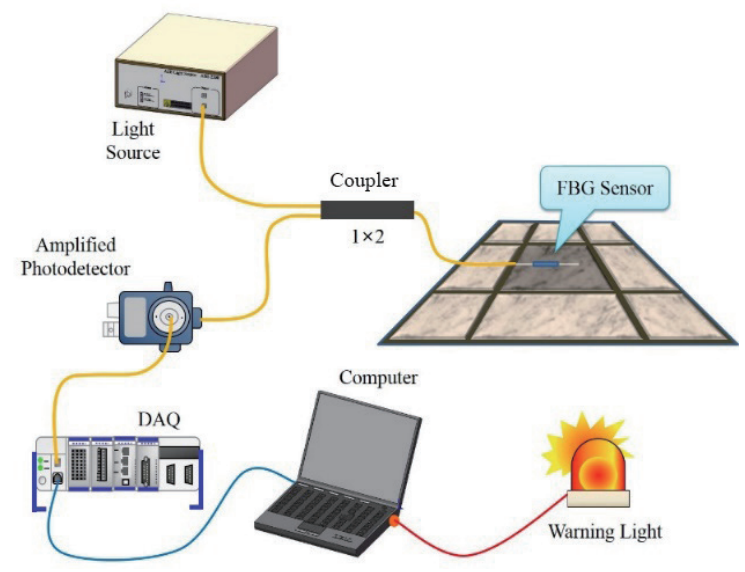

Fig. 2. (Color online) Schematic of the FBG sensor security alarm system. 

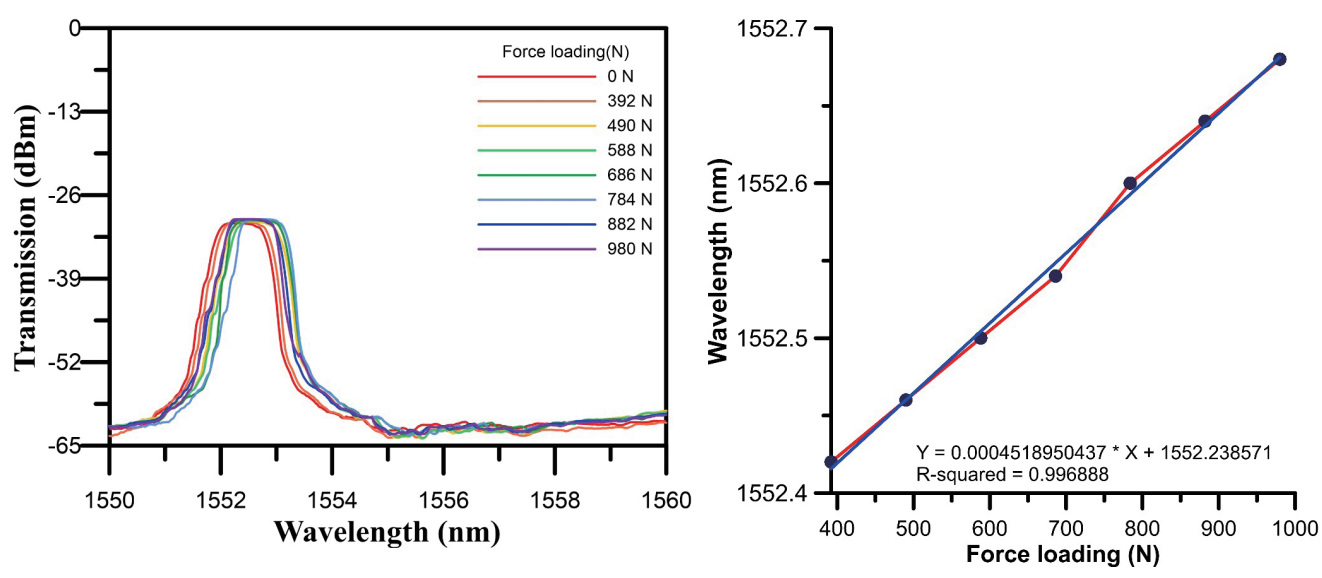

Fig. 3 (left). (Color online) Spectra of the FBG shifts with various force loadings.

Fig. 4 (right). (Color online) Relationship between force loading and wavelength shift of the FBG sensor.

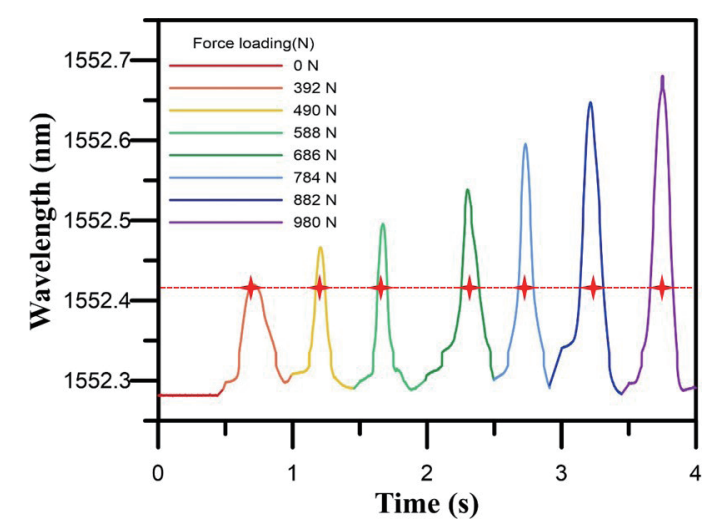

Fig. 5. (Color online) Wavelength shift of the FBG sensor that changes with various force loadings.

indicating that the FBG sensors can serve as a linear and sensitive force loading sensing device. Therefore, the experiment proved that the wavelength shift measurements can be analyzed to obtain load weights.

The loading frequency of the force loading monitoring test was $2 \mathrm{~Hz}$; in other words, the loading time interval was $0.5 \mathrm{~s}$, as loading is applied to the floor. The force loading was increased from 392 to $980 \mathrm{~N}$ in increments of $98 \mathrm{~N}$. Figure 5 shows that the wavelength shift of the FBG changes with various force loadings. We set the threshold trigger wavelength at $1552.28 \mathrm{~nm}$, indicating a $392 \mathrm{~N}$ weight loading, which is about the weight of a child, for detection. When the FBG wavelength is larger than the threshold 
trigger wavelength, the software will trigger the security alarm. In other words, if the FBG sensor wavelength moves above $1552.28 \mathrm{~nm}$, that indicates that somebody is on the floor. Moreover, values above $1552.42 \mathrm{~nm}$ indicate a human intrusion and will cause the system to set off an alarm. Intruder body weights can be determined from wavelengths between 1552.42 and $1552.68 \mathrm{~nm}$, and the resulting records can be used to create a database, so that in the future, if there is an alarm in the same band signal, we can search for related information from the database.

\section{Conclusions}

In this study, we proposed an optical fiber security system that can obtain experimental data from applied force loadings and then utilize Labview software for weight loading (security) monitoring. The following conclusions were obtained:

1. The proposed FBG sensors can provide very high sensitivity in comparison with general sensors, achieving a sensitivity of $0.000451 \mathrm{~nm} / \mathrm{N}$, which is suitable for use in an all-fiber optical security monitoring system.

2. FBG sensors are small and easy to embed in a structure for security monitoring. With PDMS packaging, the packaged FBG is robust and suitable for surface bonding on the bottom of a floor for security monitoring.

3. From the experimental results, it can be seen that as the load increases, the spectrum will shift to a longer wavelength by about $0.26 \mathrm{~nm}$. In addition, the load and the wavelength show a linear relationship, with the R-squared value reaching up to 0.996 . Thus, given the aforementioned characteristics, optical fiber security systems can provide better biometric capabilities than infrared security systems.

\section{Acknowledgements}

This work was funded by the National Science Council, Taiwan (grant number NSC100-2628-E-151-002-MY3).

\section{References}

1 K. Nerngchamnong, S. Kaviya, Y. Fujii and P. P. Yupapin: Procedia Eng. 8 (2011) 321.

2 H. Wu, Y. Rao, C. Tang, Y. Wu and Y. Gong: Sens. Actuators, A 167 (2011) 548.

3 U. Willer, M. Saraji, A. Khorsandi, P. Geiser and W. Schade: Opt. Laser Eng. 44 (2006) 699.

4 K. O. Hill, Y. Fujii, D. C. Johnson and B. S. Kawasaki: Appl. Phys. Lett. 32 (1978) 647.

5 K. Hill, B. Malo, F. Bilodeau, D. Johnson and J. Albert: Appl. Phys. Lett. 62 (1993) 1035.

6 K. Lee, K. Lee and S. Ho: WSEAS Trans. Syst. 6 (2004) 2440.

7 G. Meltz, W. W. Morey and W. Glenn: Opt. Lett. 14 (1989) 823.

8 C. S. Park, K. I. Joo, S. W. Kang and H. R. Kim: J. Opt. Soc. Korea 15 (2011) 329. 